\title{
Oral surgery: part 2. Endodontic surgery
}
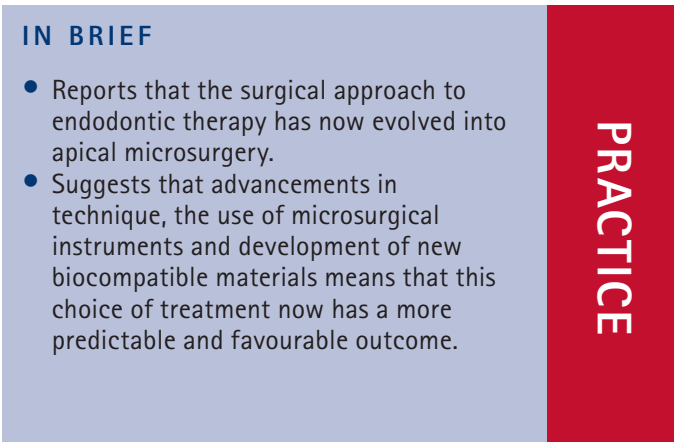

In the past, the interaction between dentoalveolar surgery and restorative dentistry has been limited to the removal of teeth with pulp and/or periradicular disease or those that were unrestorable. However, with the increasing dental awareness of the population and the retention of teeth into later life, the interaction between dentoalveolar surgery and restorative dentistry is becoming a fundamental aspect of clinical practice. Indeed, endodontic and implant surgery are core activities that facilitate the retention of a functional dentition.

\section{INTRODUCTION}

The practice of surgical endodontics in the UK has increased steadily; indeed, the number of apical surgeries performed in the general dental service in the UK has almost doubled over the past 20 years. Whether performed by a restorative, endodontic or oral surgeon, the techniques described in this paper should be within the clinical expertise of surgical dentists. The importance of interaction and co-operation between the general dentist or endodontist and the surgeon in the management of patients with persistent periradicular disease cannot be over-emphasised. Recent developments in techniques and materials for use in endodontic surgery have all been the direct result of advances in endodontology. In surgical orthodontics,

\section{ORAL SURGERY*}

\begin{tabular}{|c|c|}
\hline Part 1. & Introduction \\
\hline Part 2. & Endodontic surgery \\
\hline Part 3. & Temporomandibular disorders \\
\hline Part 4. & $\begin{array}{l}\text { Minimising and managing nerve } \\
\text { injuries and other complications }\end{array}$ \\
\hline \multicolumn{2}{|c|}{$\begin{array}{l}\text { *This series represents chapters } 1,5,7 \text { and } 8 \text { from the BDJ } \\
\text { book } A \text { clinical guide to oral diagnosis - book } 1 \text {, edited by Tara } \\
\text { Renton and C. Michael Hill. All other chapters are published } \\
\text { in the complete clinical guide available from the BDJ Books } \\
\text { online shop. }\end{array}$} \\
\hline
\end{tabular}

'Specialty doctor in Oral Surgery, King's College London Correspondence to: Ioana Pop

Email: nanatgm@yahoo.com

Accepted 16 July 2013

DOI: 10.1038/sj.bdj.2013.876

${ }^{\circledR}$ British Dental Journal 2013; 215: 279-286 where the outcome of treatment is largely predictable, the long-term management of the patient may be compromised by inappropriate inter-specialty liaison. In the practice of endodontic surgery, however, a lack of interaction will often have dramatic effects on not only the long-term but also the immediate outcome of treatment.

(Peri)apical surgery, surgical endodontics, apicectomy etc, are terms defining the surgical approach of the refractory, nonhealing periapical lesion and it should be viewed as an extension of the endodontic treatment and not as a separate entity.

Gutmann stated in 1991 that 'apical surgery intervention has emerged over the last 100 years as a significant modality in the retention of sound teeth. ${ }^{1}$ Historically there is a great deal of literature dealing with surgical $v$ s. non-surgical re-treatment endodontic procedures. Current investigations of clinical treatment attempt to use evidence-based dentistry, with prospective randomised control trials considered the highest level of evidence. Friedman et al. in 2002 suggested a rationale for non-surgical re-treatment or surgery as follows: 'For intracanal infection, non-surgical re-treatment is generally most beneficial because it seeks to eliminate the bacteria from within the root canal system. Surgery for intracanal infections can isolate but not eliminate the bacteria and would be limited to those cases where non-surgical retreatment is not judged to be possible.' ${ }^{2}$ Del Fabbro et al. in 2007 reported in a Cochrane
Database Systematic Review with quality assessment of two randomised control trials entitled Is surgical treatment more effective than non-surgical treatment when patients need re-treatment of periapical disease? ${ }^{3}$ The review concluded that there is no apparent advantage of using a surgical or non-surgical approach for the re-treatment of periapical lesions in relation to long-term outcome. The finding that healing rates can be higher for surgical cases than for nonsurgical (at least in the short term) is based on only two trials. A more recent systematic review regarding the outcomes of nonsurgical re-treatment and endodontic surgery was reported by Torabinejad et al. in 2009. ${ }^{4}$ This review was based on 26 endodontic surgery and 8 non-surgical re-treatment articles published between 1970-2008 and concluded that there is a relationship beween the follow-up interval and success with outcomes declining for surgically treated cases with increasing follow up time and the opposite was observed for the non-surgical re-treatment cases. There is currently scarce evidence for a sound decision-making process regarding alternative approaches for the re-treatment of a periradicular lesion. It is clear that there is a need for more well-designed randomised controlled trials, with follow-up periods of at least four years and a consistent sample size to detect any differences between the outcomes of the two treatments, if any.

The technology in both surgical and non-surgical approaches has advanced 
significantly in the last few years, but the challenge of eradicating micro-organisms in their biofilm is still the main reason for endodontic failure. Even with this in mind, surgical intervention is not a substitute for failure to manage the root canal system non-surgically, failure to assess thoroughly the periodontal status, and ignoring the shortcomings of the coronal seal.

Probably one of the most important aspects of the surgical approach is knowing when to choose it, as opposed to the practical experience itself. This makes the knowledge of decisionmaking as important as the expertise to be exercised in the surgical procedure. ${ }^{2}$ This is especially pertinent as Rose et al. have stated that there is a "massive and sometimes irrational move- ment to replace every endodontically treated tooth with or without symptoms with an intraosseous implant. ${ }^{5}$ However, Danin et al. mention that: 'retention of the natural tooth structure is still the goal of quality dental care and many previously root-treated teeth that appear to be done quite well but exhibit signs and symptoms are viable candidates for non-surgical, or when indicated, surgical revision of treatment. ${ }^{6}$

\section{PATIENT ASSESSMENT}

With each patient that presents for treatment, the clinician is challenged to offer choices that result in the best outcomes possible for the patient. In assessing patients for apical surgery there are a few aspects to be mentioned that influence the decision-making and the consent issues: ${ }^{7}$

- Patient complaint - whether discomfort, aesthetic or functional

- History of the present complaint previous attempts of treatment and outcomes

- Medical history - can influence the choice of treatment

- Social history - smoking can have a negative influence on the healing process

- Examination - a thorough clinical and radiological assessment can give most of the information necessary to make the treatment decision

- Formative knowledge to support the choices

- Clinical skill and experience

- Economic factors

- Evidence-based concepts

Table 1 Indications for apical surgery

\begin{tabular}{|c|c|}
\hline \multirow{9}{*}{ Anatomy } & - Calcifications of the root canals \\
\hline & - Pulp stones \\
\hline & - Canal aberrations \\
\hline & - Severe root curvature \\
\hline & - Bifurcations \\
\hline & - Secondary roots \\
\hline & - Lateral canals, delta apexes \\
\hline & - Internal and external resorption resistant to conventional tx \\
\hline & - Incomplete apex \\
\hline \multirow{5}{*}{ Procedure } & - Irretrievable, separated instruments \\
\hline & - Perforations, ledges \\
\hline & - Extruded root filling material \\
\hline & - Irremovable posts \\
\hline & - Tooth sectioning or root amputation \\
\hline \multirow{2}{*}{ Trauma } & - Root fracture \\
\hline & - Fracture of the apical third \\
\hline \multirow{2}{*}{ Biopsy } & - Suspicious and/or non-healing lesions \\
\hline & - Uncharacteristic signs and symptoms of periapical areas \\
\hline
\end{tabular}

- Patient preference after being informed fully of treatment options and their rationale

- Current best evidence and practice principles in endodontics, oral surgery and supportive disciplines.

Failure to take all factors into account may lead to treatment plans that are illadvised or not in the best interest of the patient, and therefore the consent issues are compromised. In endodontic surgery, as in any other form of surgery, it is essential that the patient is aware of the possible advantages and disadvantages of the procedure. The standard warnings given to all patients (that is, pain, swelling) should also include post-operative mobility of the root-resected teeth together with a realistic estimate of the chances of success, which varies between 50 and 90\%. In patients undergoing repeat root-resection, the increased likelihood of failure and worsened functional mobility of teeth post-operatively should be clearly explained to avoid misunderstanding and possible litigation.

Outlined below are some of the considerations regarding the indications and contraindications of surgical endodontics

\section{Pre-operative assessment of the endodontic case}

The main indications for endodontic surgery are where conventional root canal treatment has failed or where it is impossible (see Table 1). Undoubtedly, the most important points for the clinician to understand are:

1. That conventional root canal treatment should be performed in all cases where irreversible pulp inflammation or periradicular disease is diagnosed

2. The size of a periradicular lesion associated with a tooth should not influence the mode of treatment conventional root canal treatment is always the first choice of care (subject to the exclusion of more sinister pathology)

3. Poorly-performed conventional root canal treatment is not an indication for endodontic surgery (Fig. 1a)

4. The majority of patients with persistent periradicular lesions following primary root canal therapy should receive conventional root canal re-treatment, particularly when obvious deficiencies in technical quality are apparent. 


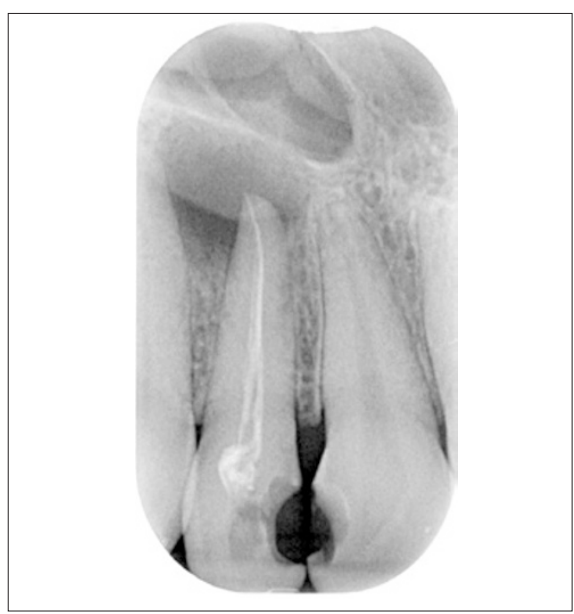

Fig. 1a Poorly performed root canal treatment

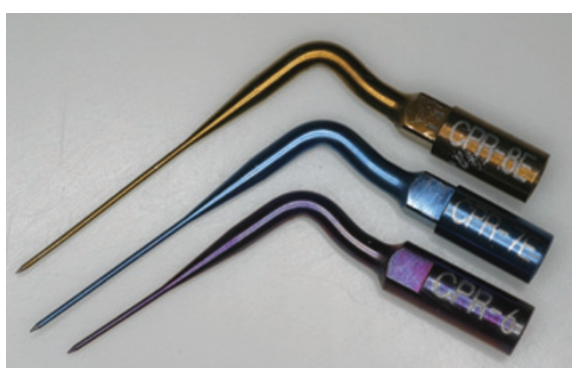

Fig. 1b Devices available for removing separated instruments and posts (CPR ultrasonic tips size 6 to 8 )

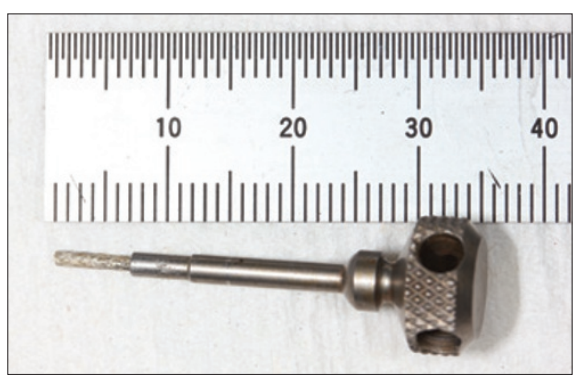

Fig. 1c Devices are available for dismantling extensive restorations and for removing posts (Ruddle post removal system)

It has been demonstrated repeatedly that well-performed conventional root canal treatment will result in a successful outcome in over $90 \%$ of cases.

Conversely, it should be borne in mind that, irrespective of the surgeon, the success rate of endodontic surgery has typically not exceeded 75\%. However, it is worth noting that recent studies of surgery performed by specialists using modern techniques and materials have reported success in over $90 \%$ of cases, but only after relatively short follow-up periods.

There are several explanations for the low success rate following surgery but the most important factor is the leakage

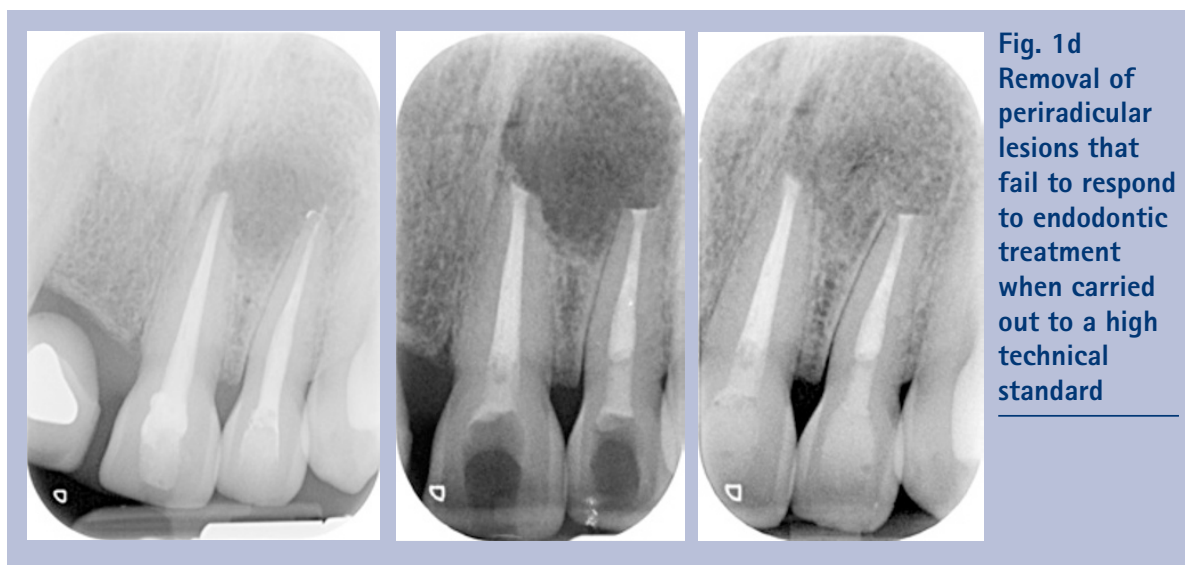

of micro-organisms or their products into the periradicular tissues. This leakage can occur past the root end filling, through cut dentinal tubules or via lateral canals. In this context, potential treatment outcomes should be explained to the patient in a clearly understandable form that allows informed decision-making. In general, a surgical approach is unlikely to remove the infection from the entire root canal system - it merely aims to contain it within the root. Conversely, conventional re-treatment adopts a more biological approach that aims to eliminate intracanal infection by debriding the canal system through irrigation and biomechanical preparation.

In planning apical surgery, operators must always bear in mind what they are trying to achieve and ask themselves three basic questions:

1. Is the tooth functionally useful?

2. Is it restorable?

3. Is its bone support adequate?

\section{Indications for surgical endodontics $^{8}$}

The surgical approach in dealing with failed endodontic cases should be carefully considered and, when feasible, root canal re-treatment must always be considered first. Furthermore it is worth mentioning that it is indicated in most cases with persistent, symptomatic, apical pathology. Some of the possible cases in which surgery is indicated are classified below:

- Inability to access or prepare the root canal due to an obstruction, post or fractured instrument. There are considerable numbers of devices and techniques available for dismantling extensive restorations and for removing separated instruments and posts, which are very effective (Figs $1 \mathrm{~b}$ and $\mathrm{c}$ )

- Inability to access or prepare the canal due to abnormal anatomy or physiological/pathological changes (for example, sclerosis, severe curvature). Although bizarre anatomical features are seen in all tooth types, there are very few occasions when a conventional approach is impossible. In addition, canals that appear occluded on radiographs are often patent when access has been achieved. In these circumstances the use of magnification is invaluable

- Removal of periradicular pathology that fails to respond to conservative treatment when carried out to a high technical standard, for example, cysts, root resorption, or extruded root material (Fig. 1d)

- Trauma; either external (eg dentoalveolar fracture) or internal (eg perforation requiring repair). However, some perforations can and should be addressed exclusively using an intra-canal approach whilst others require a combined approach.

\section{Contra-indications to surgical endodontics}

In contrast, there are cases when surgery is not indicated, ${ }^{8}$ as outlined below, and when alternative treatment should be considered as first choice (Table 2).

\section{PLANNING SURGERY}

As in any other form of dentoalveolar surgery, recent radiographs are mandatory for surgical endodontics. In assessing the images the surgeon should bear in mind the three Rs:

- Restorability (Is the tooth restorable 
and does it have a reasonable prognosis?)

- Root filling (Is it present? If present, is it satisfactory? If it is unsatisfactory - re-treat it)

- Relationship of the apex to anatomical structures (the mental nerve and maxillary sinus).

The periapical radiograph is the image most frequently employed and will usually demonstrate adequately the anatomy of the roots and canals (i.e. their number and form). However, on occasions a number of films will be required to demonstrate the complexities of the root system. Furthermore, an intimate working knowledge of canal anatomy is vital as radiographs cannot show the subtle intricacies that frequently occur in root canals. For example, there may be multiple canals within individual roots (present in 90\% of maxillary molars and $40 \%$ of mandibular incisors). Additionally, these often have bizarre communications between them, features which are often overlooked in the surgical literature.

Needless to say, all teeth should be optimally root-filled prior to endodontic surgery. This cannot be over-emphasised as it has been demonstrated in countless longterm studies that inadequate intra-canal preparation, prior to surgery, prejudices the outcome. In some instances it may be impossible to obtain a symptom-free tooth prior to surgery, necessitating the occasional use of a 'throughand through' procedure.

\section{RADIOGRAPHIC ASSESSMENT OF TEETH FOR ENDODONTIC SURGERY}

Prognosis of the tooth:

- Quality of primary root canal treatment

- Tooth tissue loss

- Root fracture

- Root resorption

- Bone support.

Planning the surgical procedure:

- Number of roots

- Root morphology

- Canal morphology

- Relationship to vital structures.

In assessing the surgical difficulty, multirooted teeth are generally more difficult

\begin{tabular}{|c|c|}
\hline \multirow{3}{*}{ Patient } & - Severe systemic disease \\
\hline & - Psychological considerations \\
\hline & - Poor oral hygiene that cannot be improved within a reasonable period \\
\hline \multirow{4}{*}{ Procedure } & - Missing or poor conventional orthograde root canal therapy \\
\hline & - Inadequate periodontal support \\
\hline & - Inadequate coronal seal \\
\hline & - Tooth subsequently unrestorable \\
\hline \multirow{3}{*}{ Anatomy } & - Lack of surgical access \\
\hline & - Unusual bony or root configuration \\
\hline & - Possible involvement of the neurovascular bundle \\
\hline Operator & - Lack of adequate skill, training or experience \\
\hline
\end{tabular}

than single-rooted teeth. This finding is principally related to surgical access, regional anatomy and canal morphology. The principles that govern the selection of anaesthesia mode (that is, local anaesthesia \pm sedation $v$ s. general anaesthesia) are as discussed in the previous paper.

\section{SURGICAL TECHNIQUE}

In gaining experience of endodontic surgery, it is most useful for the surgeon to start by operating on single rooted teeth with single canals where the access is good and the root contour is clear, that is, not mandibular incisors. The ideal teeth on which to develop suitable experience and competence are maxillary incisor teeth.

The management of patients undergoing an apical surgery procedure can be done using local anaesthesia alone, local anaesthesia and conscious sedation or general anaesthesia. The choice of the technique depends on the patient level of anxiety and the medical and clinical conditions and represents an important part of the consent procedure. The operative protocol detailed below outlines the surgical technique and the relevant modern updates found in the literature. Some of the modern approaches to surgical endodontics mentioned include the use of cone beam computer tomography in selected cases as suggested by Patel et al. ${ }^{9}$

An approximate 60\% success rate has been reported by Friedman et al. ${ }^{10}$ in 1998 when root-end resection was traditionally performed with a bevel of $45^{\circ}$ to allow visualisation of the main canal, followed by a root-end cavity preparation with a round bur. With the introduction of the intraoral microscope, root-end resection at $90^{\circ}$ and apical preparation using an ultrasonic retro tip to the depth of three to four $\mathrm{mm}$, this allowed a success rate of over $90 \%$ as demonstrated by Tsesis et al. in 2006. ${ }^{11}$ The use of the dental operating microscope has also been shown to be of benefit during surgery by Pecora et al. in $1993 .{ }^{12}$

Complete microsurgical instruments including microprobes, micromirrors, microspoon excavators and microsurgical scalpels, are essential to perform modern apical surgery, according to Johnson et al. (Figs 2 and 3). ${ }^{13}$

\section{Pre-operative protocol}

It is advisable to prescribe pre- and postoperative oral analgesics to control any degree of discomfort. According to the technique used, blood pressure measurement and administration of conscious sedation should be performed to a standard protocol and according to current guidelines. ${ }^{14}$

\section{Local anaesthetic}

The use of 2\% lignocaine with 1:80,000 adrenaline for buccal, palatal or lingual infiltrations is considered the gold standard as mentioned by Kim et al. in 1997. ${ }^{15}$ Unless contraindicated, it is mandatory to use local anaesthesia with a vasoconstrictor component in order to aid haemostasis and achieve a dry operating field.

\section{Soft tissue management}

There are several types of flap design and 


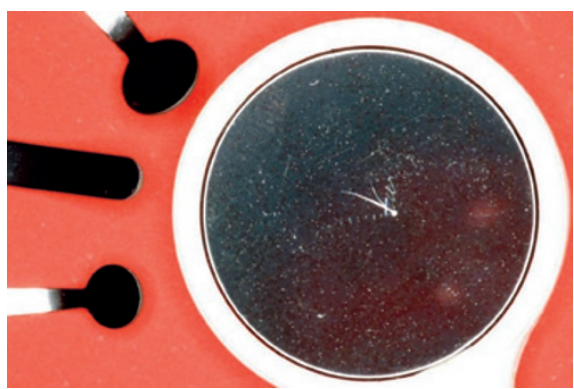

Fig. 2 Surgical micromirrors in contrast to normal size dental mirror

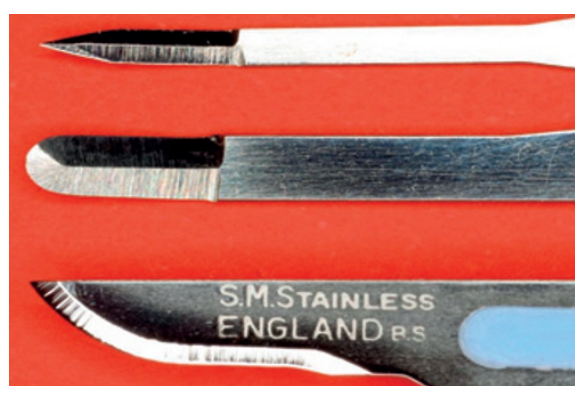

Fig. 3 Surgical microblades in contrast to normal size surgical blade
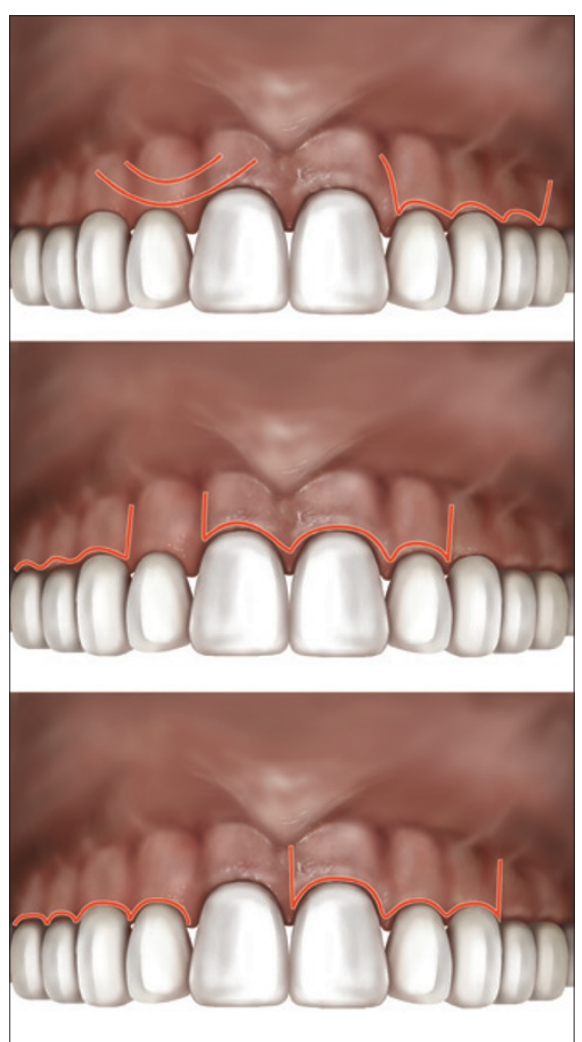

Fig. 4 Flap designs

each one of them is suited to different clinical situations. Mentioned below are those most commonly used for apical surgery:

- Full buccal/palatal mucoperiosteal

flap: two- or three-sided with mesial and/or distal relieving incisions. Involves raising the entire gingival mucosa and periosteum to gain access to the surgical site
- Sub-marginal mucoperiosteal flap (Luebke-Oschenbein): ${ }^{16}$ can be two- or three-sided with mesial and/or distal relieving incisions and preserves the gingival margin (Fig. 4)

- Papilla based incision flap: a partly full/split thickness flap suggested by Velvart to prevent papilla recession. ${ }^{17}$

The semilunar flap, which used to be very popular, is to be avoided due to an increased incidence in scarring and the lack of predictability in determining the size of the periapical lesion. ${ }^{18}$

Factors to be considered when raising a flap include the size of the periradicular lesion, periodontal status, the nature and extent of the coronal restorations, the operator choice and the proximity of significant anatomical structures such as the maxillary antrum, nasal cavity, inferior dental nerve and mental foramen.

Minimal trauma should be inflicted during incision, elevation and reflection of the tissue flap. Both reflected and unreflected tissue should be kept moist during the entire procedure, especially when a high degree of haemostasis has been achieved. Flap design plays an important role as to how much recession will occur after surgery.

\section{Hard tissue management}

- Osteotomy: access to the apical area should be obtained using a round bur in a straight slow hand piece with water irrigation. High speed handpieces should be avoided to prevent the development of surgical emphysema unless a high speed dental surgical $45^{\circ}$ handpiece is used

- Apical resection using a fissure bur at $90^{\circ}$ or with a bevel not higher than $10^{\circ}$ for better access as demonstrated by Thesis et al. ${ }^{11}$ The reasoning behind the bevel degree has also been mentioned by Gilheany et al. in 1994: "the more severe the bevel angle ( 0 to 45 degrees), the deeper the retropreparation must be to consistently produce a quality retroseal. ${ }^{19}$ Also, an angle of less than $10^{\circ}$ ensures the complete removal of a possible apical delta or lateral apical canals. Magnification and retromirrors are of immense help in diagnosing the root

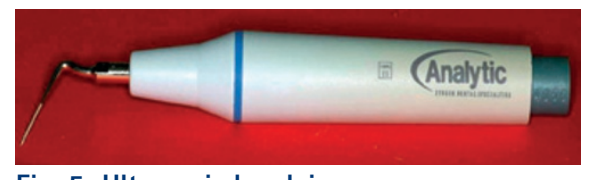

Fig. 5 Ultrasonic handpiece

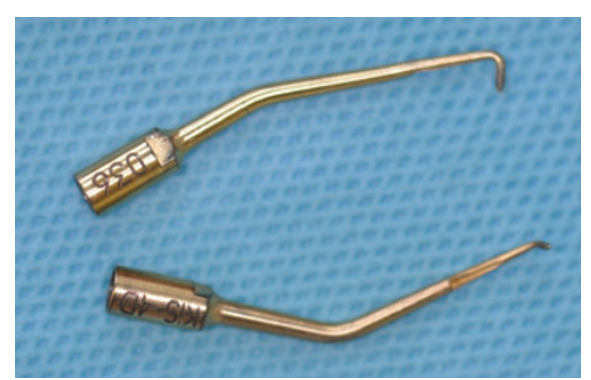

Fig. 6 Diamond coated ultrasonic retrotips (KiS tips Obtura-Spartan, Fenton, MO USA)

end variables and reduce the need to sacrifice the root length

- Curettage of the periapical pathology: the tissue removed from the periapical area should be submitted for histopathology

- Root end preparation aims to achieve a retrograde cavity with retentive or parallel walls in the long axis of the tooth in order to retain the chosen biocompatible filling material. Also its purpose is to include all the fins and anatomical isthmi and be sufficiently centred in order to maintain adequate wall thickness.

With the advent of endosonic cutting and noncutting retrotips with water cooling, the preparation technique with either a straight or mini contraangled handpiece has lost favour, according to Naito et al. ${ }^{20}$ who state that 'ultrasonic preparation improves outcome in apical surgery', with the aim of removing $3 \mathrm{~mm}$ of root filling material (Figs 5 and 6).

\section{Haemostasis}

Haemostasis is mandatory during the surgical procedure in order to maintain a dry surgical site and enhance visibility. ${ }^{8}$ It is achieved by applying pressure (gauze packs, bone wax etc.) or starting with the vasoconstrictor in the local anaesthetic and followed by small strips of non-cotton fibre gauze soaked in 1:1000 adrenaline. Other adjunctive coagulants include gelfoam, ferric sulphate etc.

\section{Root end inspection}

This is done with the aid of a microsurgical mirror under microscope magnification 
especially inspecting the buccal wall of the cavity to check for the complete removal of the gutta-percha or other filling material, or to identify cracks, root perforations etc.

Also root end inspection is advised postroot filling insertion in order to check the adaptability of the filling to the cavity walls and detection of any possible voids.

\section{Root end filling materials}

'The objective is to select a biocompatible material capable of producing a hermetic seal that prevents residual irritants and oral contaminants from exiting the root canal system and entering the periradicular tissues.' ${ }^{8}$

Numerous root end filling materials have been suggested; however, the use of guttapercha or amalgam should be discouraged due to the poor qualities of these materials. Acceptable materials used as root end fillings are zinc oxide-eugenol based materials such as Intermediate Restorative Material (IRM - Dentsply Caulk) and Super Ethoxy Benzoic Acid cement (Super EBA - S-EBS Bosworth USA), glass ionomers and composites.

However, the results of extensive studies in vitro and in vivo and positive clinical experiences makes the mineral trioxide aggregate (Pro RootTM MTA, Dentsply Tulsa Dental) the material of choice due to its biocompatibility, ability to induce hard tissue formation (bone, cementum), reduced leakage and inflammation in contact with the living tissues.

MTA is a powder consisting of fine hydrophilic particles of tricalcium silicate, tricalcium aluminate, tricalcium oxide and silicate oxide. It also contains small amounts of other mineral oxides, which modify its chemical and physical properties. Bismuth oxide powder has been added to make the aggregate radio-opaque.

The powder is mixed with water (3:1 ratio) to form a gel with a $\mathrm{pH}$ of 12.5 , which solidifies in approximately three to four hours. The MTA is applied using a carrier and condensed endosonically as suggested by Torabinejad et al. ${ }^{21} \mathrm{~A}$ dampened cotton pellet is used to clear the root tip of the excess MTA.

Another promising biocompatible rootend material is BiodentineTM (Septodont), ${ }^{22}$ a biocompatible material with a tricalcium silicate core, which has superior handling

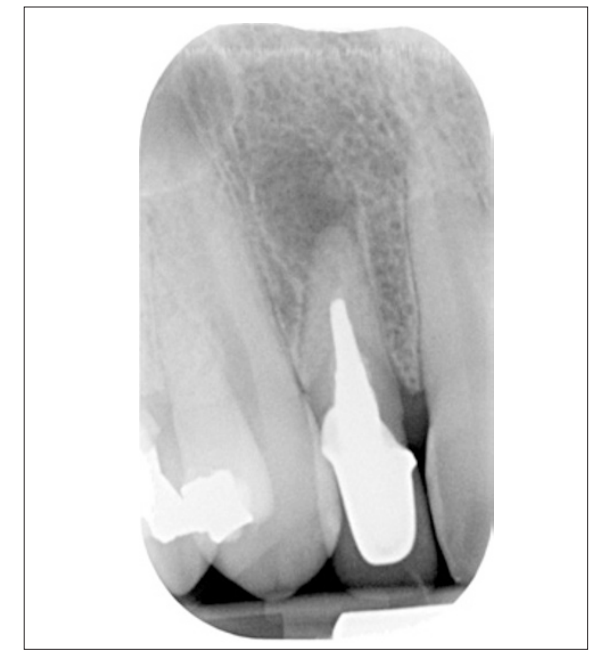

Fig. 7a Preoperative radiograph showing an upper right lateral incisor with a failed orthograde endodontic treatment and a periapical radiolucency prior to apical surgery

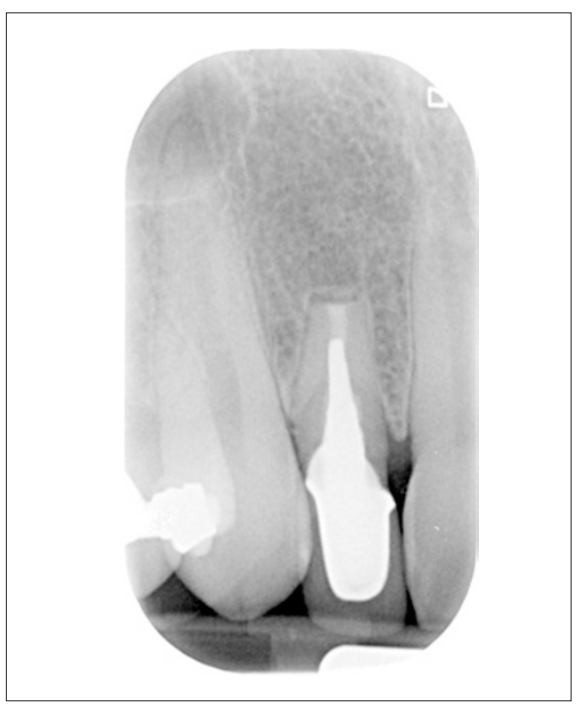

Fig. 7b Postoperative radiograph showing the same case at the one-year follow-up with a good healing outcome

properties to MTA, and exhibits similar biocompatibility. More long term followup studies are needed in order to establish firmly the qualities of this material. One of the possible drawbacks of Biodentine is its reduced radio-opacity, which makes this material difficult to assess on a post-operative radiograph.

At this stage a post-operative radiograph will help in assessing the apical restoration at the surgical site prior to the closure of the wound and will act as a reference for assessing future healing.

\section{Suture materials}

'The objective is to maintain the position of the flap reattachment by using a suture material that is strong, non-traumatic,

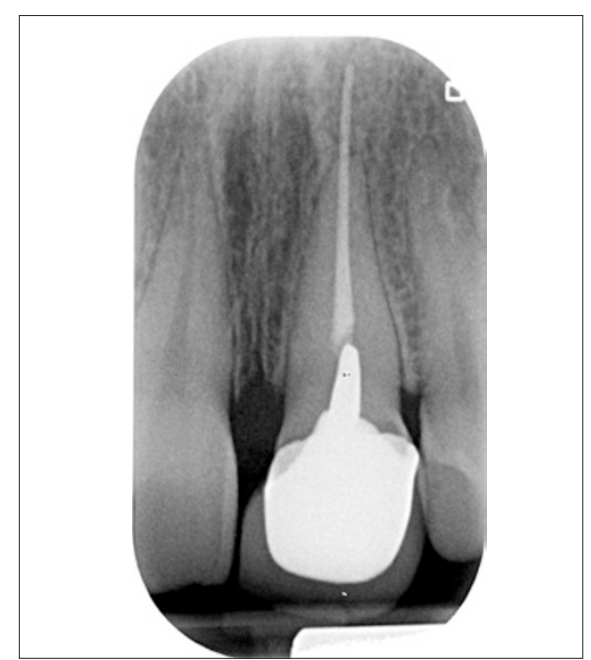

Fig. 8a Preoperative radiograph showing an upper left central incisor with an overextended root canal obturation prior to apical surgery

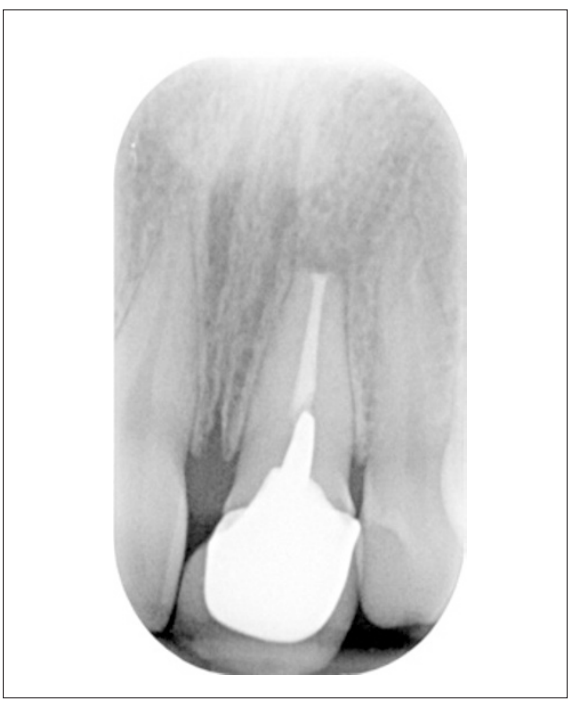

Fig. 8b Postoperative radiograph showing the same case at the one-year follow-up with a good healing outcome

non-allergenic, and easy to use.8

Suture materials can be divided in to two groups: resorbable and non-resorbable. Resorbable materials potentially cause irritation in the tissue until they are metabolised or removed. Therefore, in suturing gingival wounds, non-resorbable materials are recommended as the inflammation reaction is less and ceases after the sutures have been removed, provided they are removed within a few days, to prevent epithelial tract formation along the suture line.

Monofilament and multifilament sutures are available, and also they come in different sizes and with different surgical needles.

Modern microsurgical wound closure requires a non-absorbable suture material 


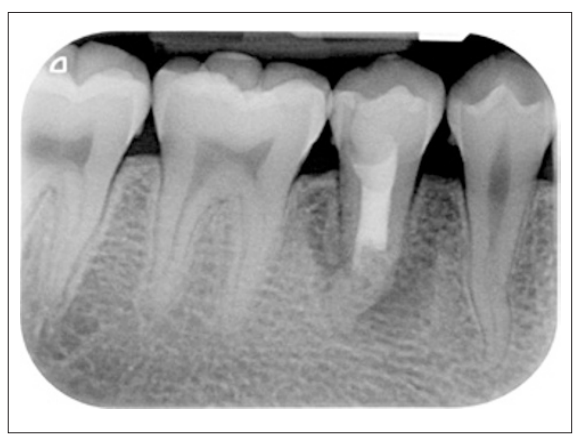

Fig. 9a Preoperative radiograph showing a lower right second premolar tooth with an apical root fracture and extensive periapical radiolucency prior to apical surgery

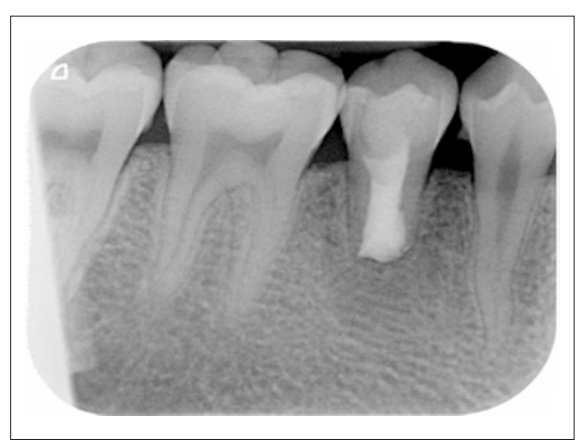

Fig. 9b Postoperative radiograph showing the same case at the one-year follow-up with a good healing outcome

in sizes 6-0 to 8-0 and is performed with the aid of a magnification device. Sutures are removed three to four days post-operatively.

\section{Recovery}

Post-operative instructions should be advised and post-operative analgesics should be prescribed if indicated for each individual case. Post-surgical infections following surgical endodontic procedures are very rare. The administration of antibiotics is seldom required and cannot be justified as part of the routine post-surgical regimen.

\section{FOLLOW-UP PROTOCOL}

An initial review appointment is required to remove sutures and assess early healing. Thereafter, regular review appointments should be made to assess healing using criteria based upon clinical and radiological examination. ${ }^{24}$

In order to avoid the limitations of conventional radiographs (anatomical noise, geometrical distortion) periapical radiographs should be taken using beam aiming devices and maintaining the same angulation.

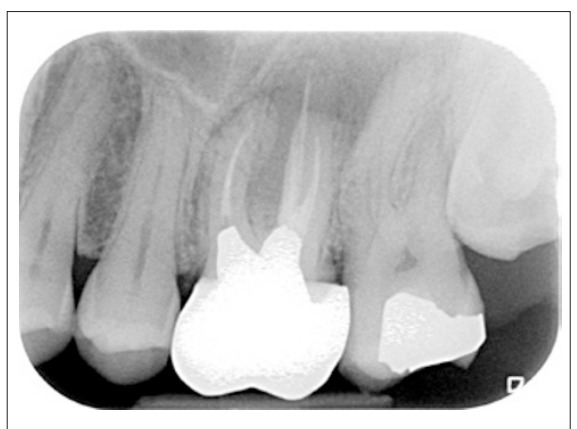

Fig. 10a Preoperative radiograph showing an upper left first molar tooth with a failed orthograde endodontic re-treatment and periapical radiolucency prior to the apical surgery of the buccal roots

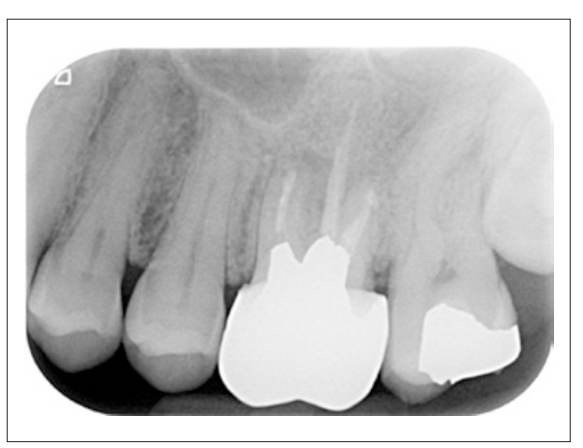

Fig. 10b Postoperative radiograph showing the same case at the one-year follow-up with a good healing outcome

\section{Long-term review}

Bony healing of the site of surgery may take many months or even years. In practical terms it is illogical to repeatedly review and re-radiograph patients following endodontic surgery. A sensible rationale, if there are no acute problems, would be to re-assess the patients (clinically and radiographically) at six months and one year following surgery.

Much has been written about measuring the outcome of apical surgery; in general there should be no clinical signs or symptoms and the radiographic appearance should demonstrate complete healing, or the presence of limited radiolucency associated with scar formation. According to Friedman, ${ }^{9}$ the success of apical surgery should be based on patient clinical factors and not solely upon radiological findings. Therefore the lesions following apical surgery can be classified as:

- Healed - no clinical signs and symptoms and no residual radiographic radiolucency. Also in this category the typical appearance of the scar is included

- Healing - no clinical symptoms and reduced radiographic appearance for a period shorter than four years

- Persistent disease - presence of clinical signs symptoms and/or persistence of radiolucency.

When failure is evident, any future treatment should be based on the identification of the cause; it is essential that the clinician evaluates such problems as root fracture and residual infection within the root canal prior to further intervention.

\section{CONCLUSION}

Clinicians should possess current knowledge about the modern techniques, prognosis and expected outcome of apical surgery.

Previous outcome studies ${ }^{2,7,19}$ have looked at pre-operative factors that can influence the prognosis of apical surgery. T. von Arx et al. in a metaanalysis of the prognostic factors in apical surgery with root-end filling concluded that "the cases associated with significant higher healing rates include the cases without pre-operative signs and symptoms, cases with good density of root canal fillings, cases with absence or size $<5 \mathrm{~mm}$ of periapical lesion and cases treated with the use of an endoscope.'23

Scarring (that is, fibrous tissue) or incomplete healing, as indicated by minimal radiographic radiolucency, which is symptom-free and non-progressive, should not be seen as failure of apical surgery.

In summary, in carefully selected cases, using a modern microsurgical approach according to the current guidelines ${ }^{24}$ the outcome of apical surgery is good, and it should become part of the treatment plan when feasible, before considering tooth extraction and replacement. Clinical cases are shown in Figures 7-10.

1. Gutmann J L, Harrison J. Surgical endodontics. Boston: Blackwell Scientific Publications, 1991.

2. Friedman D S. Considerations and concepts of case selection in the management of post-treatment endodontic disease (treatment failure). Endodontic Topics 2002; 1: 54-78.

3. Del Fabbro M, Taschieri S, Testori T, Francetti L, Weinstein R L. Surgical versus non-surgical endodontic re-treatment for periradicular lesions. Cochrane Database Syst Rev 2007; CD005511.

4. Torabinejad $M$, Corr $R$, Handysides $R$, Shabahang $S$. Outcomes of nonsurgical retreatment and endodontic surgery: a systematic review. J Endod 2009; 35: 930-937.

5. Rose L F, Weisgold A S. Teeth or implants: a 1990s dilemma. Compend Contin Educ Dent 1996; 17: 1151-1159.

6. Danin J, Stromberg T, Forsgren $H$, Linder LE, Ramskold L O. Clinical management of nonhealing periradicular pathosis. Surgery versus endodontic retreatment. Oral Surg Oral Med Oral Pathol Oral 
Radiol Endod 1996; 82: 213-217.

7. James L, Gutmann J L. Surgical endodontics: quo vadis? Endodontic Topics 2005; 11: 1-3.

8. Donald E. Practical lessons in endodontic surgery. Illinois: Quintessence Publishing Co., 1998.

9. Patel S. The use of cone beam computed tomography in the conservative management of dens invaginatus: a case report. Int Endod J 2010; 43: 707-713.

10. Friedman DS. Treatment outcome and prognosis of endodontic therapy. In Orstavik D, Pitt Ford T R (eds) Essential endodontology. pp 367-401. Oxford: Blackwell Science, 1998.

11. Tsesis I, Rosen E, Schwartz-Arad D, Fuss Z Retrospective evaluation of surgical endodontic treatment: traditional versus modern technique. J Endod 2006; 32: 412-416.

12. Pecora G, Andreana S. Use of dental operating microscope in endodontic surgery. Oral Surg Oral Med Oral Pathol 1993; 75: 751-758.
13. Johnson B R, Witherspoon D E. Surgical endodontics. St Louis: Mosby, 2006.

14. Standards in conscious sedation. DSTG, 2000. Online standards available at http://www.dstg.co.uk/ standards-in-conscious-sedation-for-dentistryl (accessed July 2013).

15. Kim S, Rethnam S. Hemostasis in endodontic microsurgery. Dent Clin North Am 1997; 41: 499-511.

16. Luebke R G. Surgical endodontics. Dent Clin North Am 1974; 18: 379-391.

17. Velvart P. Papilla base incision: a new approach to recession-free healing of the interdental papilla after endodontic surgery. Int Endod J 2002; 35: 453-460.

18. Chindia M L, Valderhaug J. Periodontal status following trapezoidal and semilunar flaps in apicectomy. East Afr Med J 1995; 72: 564-567.

19. Gilheany P A, Figdor D, Tyas M J. Apical dentin permeability and microleakage associated with root end resection and retrograde filling. J Endod 1994;
20: $22-26$.

20. Naito T. Ultrasonic preparation improves outcome in apical surgery. Evid Based Dent 2008; 9: 53.

21. Torabinejad $\mathrm{M}$, Chivian N. Clinical applications of mineral trioxide aggregate. J Endod 1999; 25: 197-205.

22. Colon P, Bronnec F, Grosgogeat B, Pradelle-Plasse N. Interaction between calcium silicate cement (Biodentine ${ }^{\circledR}$ ) and its environment. Abstract 0401 Barcelona: IADR, 2010

23. Thomas von Arx T, Penarrocha M, Jensen S. Prognostic factors in apical surgery with root-end filling: a meta-analysis. J Endod 2010; 36: 957-973.

24. Renton T, Bishop K, Glynis E. Guidelines for surgical endodontics. London: RCS, 2012. Online guidelines available http://www.rcseng.ac.uk/fds/publicationsclinical-guidelines/clinical guidelines/documents/ surgical_endodontics_2012.pdf (accessed July 2013). 